\title{
Assessment of Drug-Induced Toxicity Biomarkers in the Brain Microphysiological System (MPS) Using Targeted and Untargeted Molecular Profiling
}

\author{
Sara G. Mina ${ }^{1 \dagger}$, Begum Alaybeyoglu ${ }^{1 \dagger}$, William L. Murphy ${ }^{2}$, James A. Thomson ${ }^{3,4}$, \\ Cynthia L. Stokes ${ }^{5}$ and Murat Cirit ${ }^{1 * \neq}$
}

OPEN ACCESS

Edited by:

Yvonne Will,

Pfizer, United States

Reviewed by:

Xue Long Zhao,

University of Pennsylvania,

United States

William Mattes,

National Center for Toxicological

Research (FDA), United States

*Correspondence:

Murat Cirit

murat@javelinbio.com

${ }^{\dagger}$ These authors have contributed equally to this work

¥Present Address: Murat Cirit,

Javelin Biotech Inc., Woburn, MA, United States

Specialty section: This article was submitted to Medicine and Public Health,

a section of the journal

Frontiers in Big Data

Received: 06 February 2019 Accepted: 05 June 2019

Published: 26 June 2019

Citation:

Mina SG, Alaybeyog/u B, Murphy WL, Thomson JA, Stokes $C L$ and Cirit M (2019) Assessment of Drug-Induced Toxicity Biomarkers in the Brain Microphysiological System (MPS)

Using Targeted and Untargeted Molecular Profiling

Front. Big Data 2:23.

doi: 10.3389/fdata.2019.00023
${ }^{1}$ Department of Biological Engineering, Massachusetts Institute of Technology, Cambridge, MA, United States, ${ }^{2}$ Department of Biomedical Engineering, University of Wisconsin-Madison, Madison, WI, United States, ${ }^{3}$ Regenerative Biology, The Morgridge Institute for Research, Madison, WI, United States, ${ }^{4}$ Department of Cell and Regenerative Biology, University of Wisconsin, Madison, WI, United States, ${ }^{5}$ Stokes Consulting, Redwood City, CA, United States

Early assessment of adverse drug effects in humans is critical to avoid long-lasting harm. However, current approaches for early detection of adverse effects still lack predictive and organ-specific biomarkers to evaluate undesired responses in humans. Microphysiological systems (MPSs) are in vitro representations of human tissues and provide organ-specific translational insights for physiological processes. In this study, a brain MPS was utilized to assess molecular signatures of neurotoxic and non-neurotoxic compounds using targeted and untargeted molecular approaches. The brain MPS comprising of human embryonic stem (ES) cell-derived neural progenitor cells seeded on three-dimensional (3D), chemically defined, polyethylene glycol hydrogels was treated with the neurotoxic drug, bortezomib and the non-neurotoxic drug, tamoxifen over 14-days. Possible toxic effects were monitored with human $\mathrm{N}$-acetylaspartic acid (NAA) kinetics, which correlates the neuronal function/health and DJ-1/PARK7, an oxidative stress biomarker. Changes in NAA levels were observed as early as 2-days post-bortezomib treatment, while onset detection of oxidative stress (DJ-1) was delayed until 4-days post-treatment. Separately, the untargeted extracellular metabolomics approach revealed distinct fingerprints 2-days post-bortezomib treatment as perturbations in cysteine and glycerophospholipid metabolic pathways. These results suggest accumulation of reactive oxygen species associated with oxidative stress, and disruption of membrane structure and integrity. The NAA response was strongly correlated with changes in a subset of the detected metabolites at the same time point 2-days post-treatment. Moreover, these metabolite changes correlated strongly with DJ-1 levels measured at the later time point (4-days post-treatment). This suggests that early cellular metabolic dysfunction leads to later DJ-1 leakage and cell death, and that early measurement of this subset of metabolites could predict the later occurrence of cell death. While the approach demonstrated here provides an individual case study for proof of concept, we suggest that this approach can be extended for preclinical toxicity screening and biomarker discovery studies.

Keywords: microphysiological systems, neurotoxicity, biomarkers, metabolomics, predictive models 


\section{INTRODUCTION}

Given that central nervous system (CNS) toxicity is a leading cause of toxicity-related clinical trial failures (Cook et al., 2014; Walker et al., 2018), the predictive capabilities of current preclinical toxicity testing methods remain inadequate. The twodimensional (2D) mono-cultures of typical in vitro CNS models fail to recapitulate the physiological complexity of CNS tissues, limiting their ability to predict adverse responses at the tissue or organ level (in vivo) from the effects observed at the molecular or cellular level (in vitro) (Langhans, 2018). Further, while highthroughput screening (HTS) utilizing such 2D models enables rapid testing of large numbers of compounds, the endpoints used, such as live/dead staining and lactate dehydrogenase $(\mathrm{LDH})$ release, are necessarily simple and limited in translational relevance. Animal models remain a critical screen, yet species differences limit their predictive capability. New methodologies are sorely needed to better predict human neurotoxic liabilities of new molecular entities (NMEs) rapidly and robustly prior to clinical trials.

The emerging field of microphysiological systems (MPSs) holds promise for this mission (Marx et al., 2016; Low and Tagle, 2017). MPSs encompass a range of cellular- and tissuelevel models in three-dimensional (3D) culture platforms meant to recapitulate more physiologically-relevant functions of human organs and tissues compared to traditional 2D culture systems. MPSs are more cost-effective than animal models and can be used for numerous pharmaceutical development applications including drug absorption, distribution, metabolism, excretion, and toxicity (ADMET), evaluating efficacy and investigating pharmacodynamic mechanisms. For example, neural toxicity has been investigated in a brain MPS comprising a 3D construct of mixed neuronal and glial cells derived from human embryonic stem (ES) cells (Schwartz et al., 2015), and hepatic drug metabolism has been studied in a 3D human liver MPS (Tsamandouras et al., 2016). Likewise, drug absorption and metabolism processes have been studied in an integrated gut-liver platform that enables observation of organ-organ crosstalk (Chen et al., 2017; Tsamandouras et al., 2017). Higher degree multi-MPS systems have also been applied to assess systemic drug effects on human physiology (Maschmeyer et al., 2015; Oleaga et al., 2016; Zhang et al., 2017; Edington et al., 2018).

MPS technologies enable longer-term tissue culture to study kinetics of drug-physiology interactions using various continuous and endpoint metrics. Not only might they be used to screen out toxic molecules at the preclinical stage, but they could potentially provide insights for development and validation of clinical biomarkers. For example, omics-profiling (proteomics, metabolomics) combined with measurements of neuronal electrical activity in a mixed neuronal/glial cell culture platform identified several potential biomarkers of druginduced neurotoxicity (Schultz et al., 2015). Early response biomarkers in humans that presage later overt toxicity would be particularly valuable. As of 2011, fewer than 100 biomarkers were validated for clinical use, highlighting the difficulties connected with translating scientific findings to clinical decision making (Poste, 2011).
Fluid-based molecular biomarkers such as those found in serum, plasma, urine, and cerebrospinal fluid (CSF) have the advantages of relative ease of sampling using minimally invasive methods, as well as the possibility of frequent or even continuous monitoring. Two biomarkers of neural function accessible in blood and urine include $\mathrm{N}$-acetylaspartic acid or $\mathrm{N}$-acetylaspartate (NAA) and DJ-1/PARK7 protein. NAA, the acetylated form of the amino acid aspartate, is one of the most abundant brain metabolites (Moffett et al., 2012), and has been shown to be an indicator of neural cell function, while human DJ-1, an oxidative stress indicator, is utilized for evaluating druginduced neurotoxicity (Kahle et al., 2009).

Numerous studies have demonstrated that NAA levels are altered in the brain in a variety of human CNS disorders. Its concentration declines in nearly all, including Alzheimer's disease (Bittner et al., 2013; Murray et al., 2014), MS (Tortorella et al., 2011), and schizophrenia (Harris et al., 2006), although it is elevated in Canavan disease (Wittsack et al., 1996; Moffett et al., 2012) due to the lack of a metabolizing enzyme. NAA levels were increased in the serum of ALS patients, thought to be related to greater excretion of NAA into the circulation following release from damaged neurons (Simone et al., 2011).

DJ-1 is a ubiquitous redox-responsive protein, mainly localized in the cytosol and also found in mitochondria and the nucleus. Studies have shown that during oxidative stress, DJ-1 may modulate the expression of genes such as glutamate-cysteine ligase, which results in glutathione (GSH) metabolite formation (Kim et al., 2012). Biochemically, DJ-1 is easily oxidized in response to several oxidative stimuli, and the oxidized, acidic isoforms of DJ-1 have been found to be accumulated in the brains of patients with sporadic Parkinson disease and Alzheimer's disease (Choi et al., 2006).

In this study, drug-induced neurotoxicity using targeted and untargeted biomarkers was evaluated in the brain MPS. The effects of the chemotherapeutic compound bortezomib, a proteasome inhibitor and known neurotoxic drug (Badros et al., 2007; Argyriou et al., 2014; Canta et al., 2015), were assessed and compared to effects of the selective estrogen receptor modulator (SERM) tamoxifen, which has no clinically reported neural adverse effects (Ernst et al., 2002; Stouten-Kemperman et al., 2015; Hong et al., 2017). Neurotoxicity was first assessed with the targeted biomarkers NAA and DJ-1 protein. Then, untargeted metabolomics was used to identify additional early response biomarkers after the first drug dose and these were correlated with NAA and DJ-1 at different time points to identify whether they are predictive of known markers of neuronal function and toxicity measured at later times.

\section{MATERIALS AND METHODS}

\section{Neural Progenitor Cell Culture}

Neural progenitor cells (NPCs) derived from the human H1 ES line developed in the laboratory of Professor James Thomson, Morgridge Institute, Madison, WI were provided for this study. The NPC derivation methods were previously described (Schwartz et al., 2015). NPCs were cultured at $37^{\circ} \mathrm{C}$ and $5 \% \mathrm{CO}_{2}$ in neural expansion medium comprising DMEM/F-12 medium 
$(500 \mathrm{~mL})$ supplemented with $\mathrm{rhFGF} 2(5 \mathrm{ng} / \mathrm{ml}), 1 \mathrm{X}$ N2 (Life Technologies, Carlsbad, CA, USA), 1X B27 (Life Technologies), L-ascorbic acid-2-phosphate magnesium (64 mg/L, SigmaAldrich, St. Louis, MO, USA), Sodium/selenium (14 $\mu \mathrm{g} / \mathrm{L}$, Sigma), NaHCO3 (543 mg/L, Sigma), penicillin-streptomycin (10\% v/v 10,000 units/ml, ThermoFisher, Waltham, MA, USA). Cryopreserved NPCs were passaged on T-75 flasks coated with Matrigel (growth factor reduced, Corning 356230, $0.1 \mathrm{mg} / \mathrm{ml}$ for $1 \mathrm{~h}$ ) in neural expansion medium. NPCs were maintained in a humidified incubator at $37^{\circ} \mathrm{C}$ and $5 \% \mathrm{CO}_{2}$ below $90 \%$ confluence on Matrigel-coated polystyrene flask before passage. Cells were passaged every 4-5 days using Corning Accutase Cell Detachment Solution, Liquid (ITC AT104, Mediatech, Manassas, VA, USA) and neural expansion medium to neutralize Accutase. Cells were then pelleted by centrifugation at 240 relative centrifugal force for $3 \mathrm{~min}$ and a cell count was performed using the Luna Countess ${ }^{\mathrm{TM}}$ II FL Automated Cell Counter (ThermoFisher Scientific, Waltham, MA, USA). NPCs were cryopreserved at $1.2 \times 10^{7}$ cells per vial or harvested on MPS.

\section{Polyethylene Glycol (PEG) Hydrogel Formulation}

Polyethylene glycol (PEG) hydrogels were formed using "thiolene" photopolymerization chemistry from previously published protocols (Fairbanks et al., 2014; Hansen et al., 2014). The PEG hydrogel solution was purchased from Stem Pharm, Inc. (Madison, WI, USA). Hydrogel formulation comprised 40 $\mathrm{mg} / \mathrm{mL}$ 8-arm PEG-norbornene, 4.8 MMP-peptide crosslinker (9.6 M cysteine, $60 \%$ molar ration relative to norbornene arms), 2 mM CRGDS C-amidated peptide, and Irgacure 2959 photoinitiator in PBS. Gels were polymerized in $0.33 \mathrm{~cm}^{2}$ Transwell ${ }^{\circledR}$ inserts (Corning 3470, Corning Life Sciences, Teterboro, NJ, USA) with $40 \mu \mathrm{L}$ total volume and $4.8 \mathrm{~J} / \mathrm{cm}^{2}$ of $365 \mathrm{~nm}$ UV light (10 $\left.\mathrm{min}, 8 \mathrm{~mW} / \mathrm{cm}^{2}\right)$.

\section{Drug Preparation}

Lyophilized drugs were solubilized in dimethyl sulfoxide (DMSO) and drug stock solutions were prepared in neural expansion medium. Bortezomib (Sigma-Aldrich) was prepared in the dark by dissolving powder in $100 \%$ DMSO and sterile filtered to obtain a stock solution of $10 \mathrm{mM}$. $0.1 \%$ drug stock was serially diluted in media for final dosing concentrations ranging from 0.001 to $10 \mu \mathrm{M}$, increasing by factors of ten. Tamoxifen (LC Laboratories, Woburn, MA, USA) was prepared by dissolving powder in $100 \%$ DMSO and sterile filtered to obtain a stock solution of $10 \mathrm{mM}$. Drug stock was serially diluted in media for final dosing concentrations ranging from 0.01 to $10 \mu \mathrm{M}$, increasing by factors of ten.

\section{Formation of 3D Neural Constructs and Neurotoxicity Experiments Seeding H1 ES Cell-Derived Neural Progenitor Cells (NPCs) on PEG Hydrogels}

The NPCs were harvested for MPS seeding between passages 5 and 8 . NPCs were seeded at density of 50,000 cells $/ 0.33 \mathrm{~cm}^{2}$ Transwell ${ }^{\circledR}$ and cultured on the PEG gel for 14-days prior to drug exposure. NPC media was changed every 2-days using $200 \mu \mathrm{L}$ in the apical compartment, $1 \mathrm{~mL}$ in the basal compartment.

\section{Drug-Induced Neurotoxicity Experiments}

After 14-days of culture to establish the brain MPS, the 3D neural constructs were exposed to chemotherapeutic compounds for another 14-days, with media exchanged every 2-days. The time reported for all results are relative to establishment of culture on day 0 . The chemotherapeutic neurotoxic compound, proteasome inhibitor, bortezomib (low-dose: $0.001 \mu \mathrm{M}$, middose: $0.01 \mu \mathrm{M}$, and high-dose: $0.1 \mu \mathrm{M}$ ), or non-neurotoxic compound, selective estrogen receptor modulator (SERM), tamoxifen (low-dose: $0.01 \mu \mathrm{M}$, mid-dose: $0.1 \mu \mathrm{M}$, and high-dose: $1 \mu \mathrm{M})$ were compared to untreated controls for 14-days. For context, the bortezomib clinical maximum concentration $\left(\mathrm{C}_{\max }\right)$ is $\sim 0.6 \mu \mathrm{M}$ (Moreau et al., 2012) and tamoxifen $\mathrm{C}_{\max }$ is $\sim 0.2 \mu \mathrm{M}$ (Kisanga et al., 2004). The prepared drug concentrations in the neural expansion media were added to both the apical and basal compartments to avoid concentration gradients.

\section{Quantification of NAA and DJ-1/PARK7}

$\mathrm{N}$-acetylaspartate (NAA) concentration in media samples was measured and analyzed every 2-days as described previously (Edington et al., 2018).

Human DJ-1/PARK7 concentration was measured in media samples every 2-days using an electrochemiluminescence multiarray immunoassay run according to the manufacturer's protocol (Human DJ-1/PARK7 Kit, Meso Scale Diagnostics, LLC, Rockville, MD, USA) as follows. A 7-point calibration curve with 4 -fold serial dilution steps and a zero calibrator blank were prepared in diluent buffer (Diluent 35). Media samples were thawed and diluted in media blanks $(1: 2 \mathrm{v} / \mathrm{v}$ samples/media). Plates were blocked, washed, and incubated with diluted samples then read on a MESO QuickPlex SQ 120 instrument. Data were analyzed with Meso Scale Diagnostics' Discovery Workbench software.

\section{Immunocytochemistry}

Both 2D laminin-coated Transwell inserts and 3D neural constructs on Transwell inserts were fixed in situ with 4\% paraformaldehyde (Sigma-Aldrich, St. Louis, MO, USA) and incubated for $15 \mathrm{~min}$ at room temperature. The samples were stained for neuron-specific $\beta$-III tubulin antibody $(1: 500 \mathrm{v} / \mathrm{v}$, monoclonal mouse IgG; R\&D Systems, Minneapolis, MN, USA) and glial fibrillary acidic protein (GFAP; 1:500 v/v, goat polyclonal to GFAP, Abcam, Cambridge, MA) in incubation buffer $(0.05 \%$ Triton X-100 and $1 \%$ bovine serum albumin in phosphate buffered saline), followed by overnight incubation at $4^{\circ} \mathrm{C}$. Then, the cells were washed 2 times for $60 \mathrm{~min}$ with rinse buffer $(0.05 \%$ Triton X-100 in phosphate buffer saline), followed by an overnight rinse at $4^{\circ} \mathrm{C}$ in rinse buffer. After rinse, 1:200 dilutions of secondary antibodies (Alexa Fluor ${ }^{\circledR} 488$, Alexa Fluor ${ }^{\circledR}$ 568, and Hoechst 1:1000 v/v, Invitrogen, Carlsbad, California, USA) were added, followed by incubation overnight at $4^{\circ} \mathrm{C}$ or at least $4 \mathrm{~h}$ in the dark at room temperature. After that, the cells were rinsed and incubated overnight at $4^{\circ} \mathrm{C}$ in 
rinse buffer. Fluorescent images were obtained using a Keyence confocal imaging system with a 20x objective.

\section{Measurement of Untargeted Metabolomics for Drug-Induced Neurotoxicity}

Media samples at day 16 (2-days post-treatment) for cells treated with $0.001-0.1 \mu \mathrm{M}$ bortezomib, and $0.01-1 \mu \mathrm{M}$ tamoxifen as well as untreated control were shipped for analysis to Human Metabolome Technologies America, Inc. (HMT; Boston, MA, USA). For sample preparation, $40 \mu \mathrm{L}$ of samples were mixed with $10 \mu \mathrm{L}$ of Milli-Q water containing internal standards $(1,000 \mu \mathrm{M})$. The mixture was then filtered through a $5 \mathrm{kDa}$ cut-off filter (ULTRAFREE-MC-PLHCC, Human Metabolome Technologies, Yamagata, Japan) to remove macromolecules. Metabolome analysis was performed in samples of culture medium using Capillary Electrophoresis Time-of-Flight Mass Spectrometry (CE-TOFMS) in two modes for cationic and anionic metabolites. On the basis of HMT's standard library, 65 metabolites (47 metabolites in Cation mode and 18 metabolites in Anion mode) were detected (dataset provided in Supplementary Materials).

\section{Analysis of Biomarker and Untargeted Metabolomics Data}

The measured DJ-1 and NAA levels are reported as mean \pm SD. The data were analyzed using a two-way ANOVA with Bonferroni's post-hoc testing for $n=3, p<0.05$ using GraphPad Prism version 7 (San Diego, CA, USA). For analysis of untargeted metabolomics, MetaboAnalyst R package (Chong et al., 2018) and Ingenuity Pathways Analysis (IPA, QIAGEN Bioinformatics, Redwood City, CA, USA) (Krämer et al., 2014) were used for multivariate and cluster analysis, and functional and pathway analysis, respectively. Reported metabolomics relative peak area data were normalized via auto scaling prior to statistical analysis. Altered metabolites were analyzed using multiple $t$ tests (individual $t$-tests for each metabolite) with Holm-Sidak correction and plotted using GraphPad Prism. Pathway maps were constructed in PathVisio desktop (Kutmon et al., 2015) for visualization of connectivity of the detected metabolites.

\section{Biomarker-Metabolite Correlation Analysis and Regression Models}

Prior to correlation analysis, untargeted metabolomics data was refined by removing the metabolites that were not detected (N.D.) in the majority of the samples. For those metabolites that were either not detectable or became detectable upon drug exposure, relative abundance values were assigned as 2 -fold lower than the lower limit of detection (LLOD/2). Lower limit of detection was defined as the lowest relative abundance value measured in any metabolite across the samples. Preprocessed untargeted metabolomics data was used as the input for all correlation analyses and regression models. Biomarkermetabolite correlation analyses were carried out and plotted using GraphPad Prism. Correlations with a coefficient larger than $0.7(|r|>0.7)$ were considered strong correlations and simple linear regression (SLR) models were employed for those correlated metabolite-biomarker couples. The regularized regression method Lasso (Tibshirani, 1996) was employed using the caret (Kuhn, 2008) and glmnet R packages (Friedman et al., 2010) to select the important metabolites (predictors from the intercorrelated metabolomics data; multicollinearity) to predict DJ-1 (dependent variable). The hyperparameter lambda $(\lambda)$ was optimized at the minimum root mean square error $\left(\mathrm{RMSE}_{\min }\right)$ of the repeated 5-fold cross-validations (100 repetitions) over the whole dataset ( $\mathrm{R}$ script in Supplementary Materials). The final model for prediction was constructed using the regression coefficients calculated at the optimized lambda. The predicted DJ-1 levels were then compared with the measured DJ-1 levels to calculate to goodness of fit $\left(R^{2}\right)$.

\section{RESULTS}

\section{Brain Microphysiological System (MPS) Supports Mixed Neural Cell Culture for 28-Days}

The brain MPS comprises a 3D PEG hydrogel with NPCs that have differentiated into $\beta I I I-t u b u l i n{ }^{+}$(neurons) and $\mathrm{GFAP}^{+}$ (glial) cells and self-assembled to form 3D neural constructs. The mixed neuron-glial cell culture can be maintained in this environment for at least 28-days, as demonstrated by the immunofluorescence images in Figure 1A at day 14 and Figure 1B at day 28. Structurally, the images illustrate cells extending around the circumference of the $3 \mathrm{D}$ hydrogels.

\section{Assessment of Brain MPS Response to Bortezomib Using Targeted Biomarkers}

During exposure to bortezomib from days 14-28, NAA and DJ-1 levels were measured every 2-days to assess the druginduced neurotoxicity in the brain MPS. Low-dose $(0.001 \mu \mathrm{M})$ bortezomib induced no significant difference in NAA and DJ1 levels compared to the untreated controls, as shown in Figures 2A,B. For $0.01 \mu \mathrm{M}$ dose (mid-dose) of bortezomib on day 18 (4-day treatment), NAA levels were significantly reduced compared to control, and remained so at day 22 and beyond. In contrast, DJ-1 levels significantly increased and remained elevated for the duration of the drug exposure (Figures 2C,D). These results suggest that $0.01 \mu \mathrm{M}$ bortezomib exposure leads to neurotoxicity in the brain MPS.

For high-dose $(0.1 \mu \mathrm{M})$ bortezomib, on day 16 (2-day treatment) NAA levels were upregulated compared to the untreated control (Figure 2E), falling below control at day 18 , and from day 20 (6-day treatment) through last day of treatment, falling below the limit of quantification, suggesting acute neuronal damage in that time period. In comparison, DJ1 levels were at first unaltered, but then increased sharply by day 18 , coinciding with the decline in NAA, and subsequently DJ-1 significantly decreased for the duration of treatment, compared to untreated controls, as shown in Figure 2F. Measurements and $t$-test results of bortezomib-treated brain MPSs are tabulated in Supplementary Table 1.These results demonstrated that changes in NAA and DJ-1, clinically-relevant biomarkers, in the brain MPS upon neurotoxic drug treatment were dose- and time-dependent. 


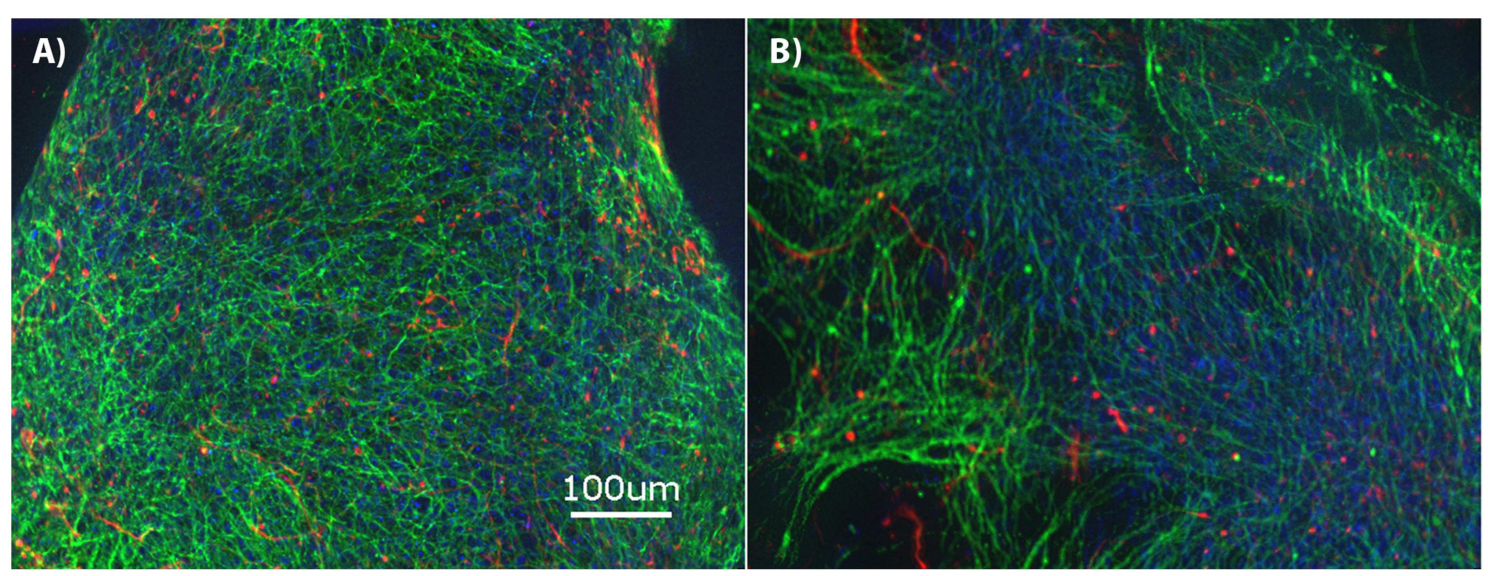

FIGURE 1 | Brain microphysiological system (MPS): Neural progenitor cells (NPCs) cultured on three-dimensional (3D) chemically-defined poly(ethylene glycol) hydrogel (multilayer) at (A) 14-days and (B) 28-days. Immunocytochemistry images for $\beta$-III tubulin (neurons; green), Glial Fibrillary Acidic Protein (GFAP: glial cells; red), and Hoechst (nuclei; blue). Scale bar $=100 \mu \mathrm{m}$.

\section{Assessment of Brain MPS Response to Tamoxifen Using Targeted Biomarkers}

The effects of tamoxifen, a SERM with no clinically observed neurotoxicity in humans, on NAA and DJ-1 levels were tested in the brain MPS between days 14-28 in the brain MPS. For $0.1 \mu \mathrm{M}$ (low-dose) and $0.01 \mu \mathrm{M}$ (mid-dose) treatments, NAA and DJ1 levels were not significantly different compared to untreated controls at any time point during treatment (Figures 3A-D). For $1 \mu \mathrm{M}$ (high-dose) tamoxifen exposure, NAA levels were slightly reduced at days 26 and 28 (after 12- and 14-day drug exposure, respectively), as shown in Figure 3E. In comparison, DJ-1 level was elevated after 4-days of drug exposure (day 18), fell below untreated control at day 20 , and then returned to levels similar to untreated control for the duration of the experiment (Figure 3F). In summary, the effects of tamoxifen on NAA and DJ-1 levels in the brain MPS are limited in comparison to those observed for bortezomib. Measurements and $t$-test results of DJ-1 and NAA levels of tamoxifen-treated brain MPSs are tabulated in Supplementary Table 1.

\section{Bortezomib and Tamoxifen Treatments Induce Distinct Metabolic Biomarker Profiles in the Brain MPS}

The untargeted metabolomics of extracellular media samples were investigated 2-days post-treatment for bortezomib and tamoxifen, and were compared to untreated controls. A total of 65 metabolites were detected in the media samples, and relative abundance values were used to indicate drug-induced alterations in the metabolic fingerprints. For the multivariate analysis, partial least squares discriminant analysis (PLS-DA) was carried out to visualize differences among the groups with respect to untreated controls. The PLS-DA scores plots revealed the separation between the different dose treatments of bortezomib (Figure 4A) or tamoxifen (Figure 4B) compared to untreated controls. Bortezomib treatment induced a more pronounced dose-dependent deviation from the untreated controls than did tamoxifen treatment, as indicated by data more closely packed and confidence regions overlapping in comparison with the untreated controls for tamoxifen. 10-fold cross validation for both PLS-DA models showed that $Q^{2}$ was 0.79 for the first 2 components of the bortezomib model with $R^{2}$ of 0.97 (Supplementary Table 2). For the tamoxifen model, calculated $Q^{2}$ values were negative for up to 8 components, suggesting that the model is either not predictive or is overfitted (Supplementary Table 2).

The relative metabolite levels in the untreated controls and the drug-treated neural constructs are compared in the heatmap (Figure 4C) to detect the metabolites altered by drug treatment. The group averages ( $n=3$ for each drug and dosing) of the metabolic profiles suggested major differences in some metabolite levels in the mid-dose and high-dose bortezomibtreated groups compared to the untreated controls, while the lowdose group appeared comparable to the untreated group. In the mid-dose and high-dose tamoxifen-treated groups, alterations in some amino acid levels were detected, but the changes were not statistically significant (not shown). The low-dose tamoxifen treated group was similar to untreated controls.

To assess the significance of altered metabolites upon bortezomib or tamoxifen treatments, statistical analysis (multiple $t$-tests) was carried out. None of the metabolite alterations in the low-dose bortezomib treated group compared to untreated control were statistically significant, while perturbations in the levels of 3 metabolites in the mid-dose treated group, and of 5 metabolites in the high-dose treated group were statistically significant $\left(p_{\text {adj }}<0.05\right)$ compared to the untreated controls. For the tamoxifen-treated groups, none of the metabolite alterations were statistically significant.

The most significant differences induced with bortezomib treatment were in the relative levels of S-sulfocysteine (SSC) and phosphorylcholine (ChoP) (Figure 5), which are the top2 metabolites with respect to the calculated VIP scores 
A

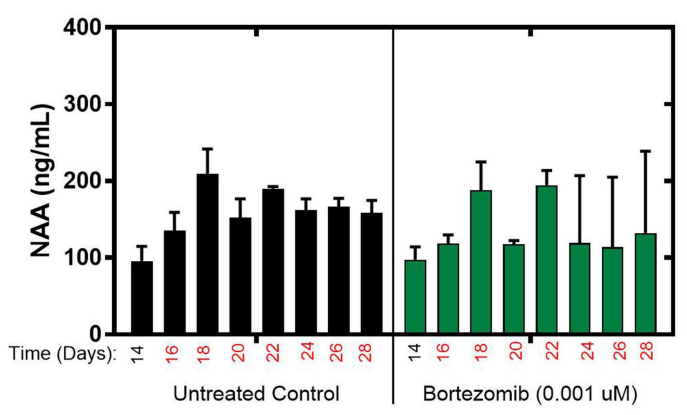

C
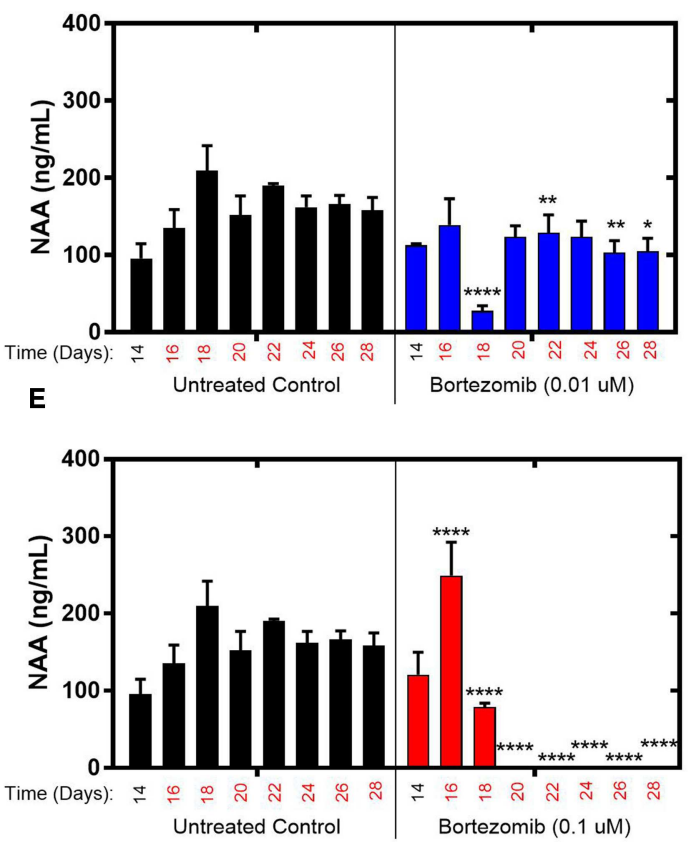

B

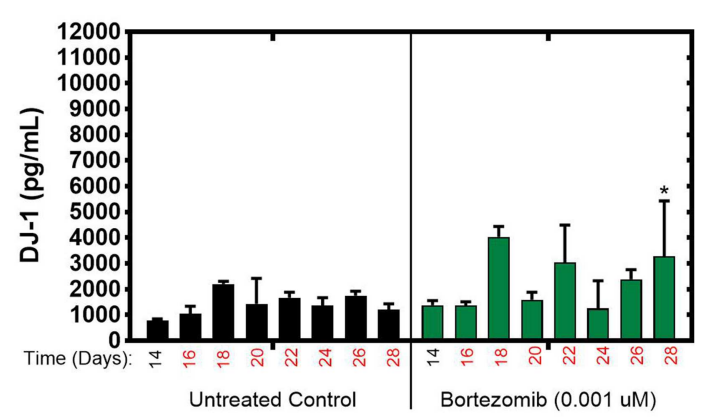

D
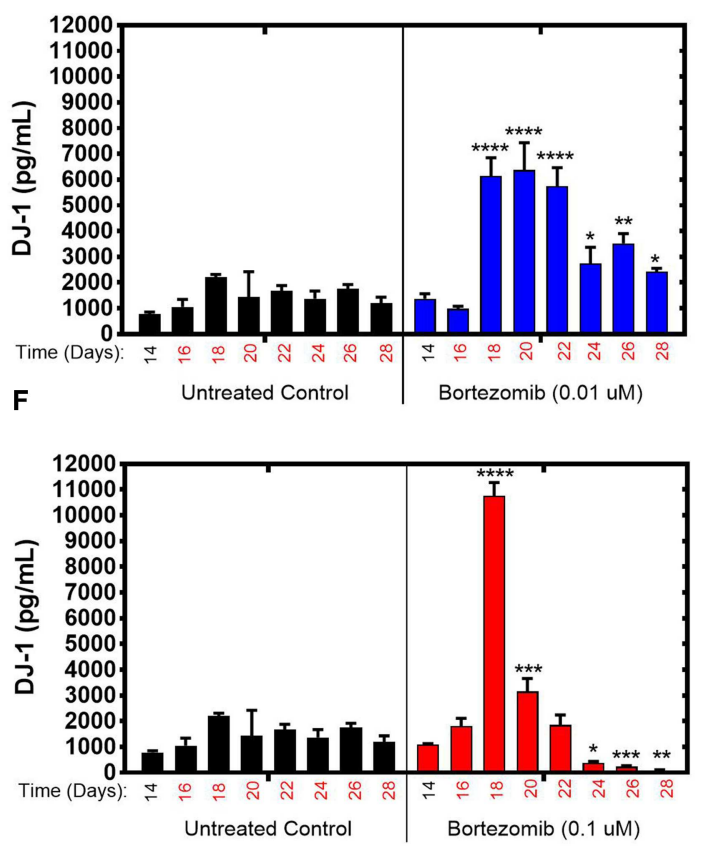

FIGURE 2 | Bortezomib treatment for 14-days in the brain MPS. (A,C,E) Extracellular N-acetyl-aspartate (NAA) concentration in the media for the noted dose (B,D,F) Extracellular DJ-1/PARK7 concentration in the media for the noted dose. Each bar represents mean \pm SD for triplicate MPS wells $(n=3)$ at each treatment dose and time point. For each bortezomib dose, significant differences between treated (colored bars) and untreated control (black bars) at the same time point are denoted according to the calculated $p$-values (two-way ANOVA multiple comparisons with a Bonferroni's post-hoc test; ${ }^{\star} p<0.05$, ${ }^{* \star} p<0.01$, ${ }^{\star \star \star} p<0.001$, and ${ }^{\star \star \star \star \star} p<$ 0.0001). Day 14 represents the time at which drug dosing was initiated.

(metabolites with VIP $>1$ were considered as the greatest contributors to the group separation) in the PLS-DA model (Supplementary Table 2). Bortezomib had a dose-dependent effect in the levels of SSC and ChoP, whereas these metabolites were unaltered upon tamoxifen treatment. Cystine, the oxidized form of cysteine, was similarly affected by bortezomib treatment, but unaltered by tamoxifen at any dose level. Although these metabolites are on the same canonical metabolic pathway, different dependencies on bortezomib dose were observed. SSC levels gradually decreased with increasing bortezomib concentration, whereas cystine levels were comparable in the untreated, low-dose, and mid-dose treated groups, but significantly lower in the high-dose bortezomib-treated group (Figure 5). Associated with ChoP, ethanolamine phosphate (PE) levels were higher in bortezomib-treated groups, but remained unaltered in tamoxifen-treated groups.

Significant changes were also detected in the levels of lactic acid, pyruvate, citric acid, and hypoxanthine upon bortezomib treatment, but unaltered in the tamoxifen-treated groups (Figure 5). An increase in extracellular levels of lactic acid was observed in the mid-dose bortezomib treated group and the levels in the high-dose bortezomib treated group were similar to that in the untreated controls. Pyruvate levels were comparable in the untreated, low-dose, and mid-dose treated groups, but significantly higher in the high-dose bortezomib treated group. A similar effect on the levels of hypoxanthine, was found, with significantly higher levels in the high-dose bortezomib treated group. Citric acid showed a strong dose-dependent trend to 
A

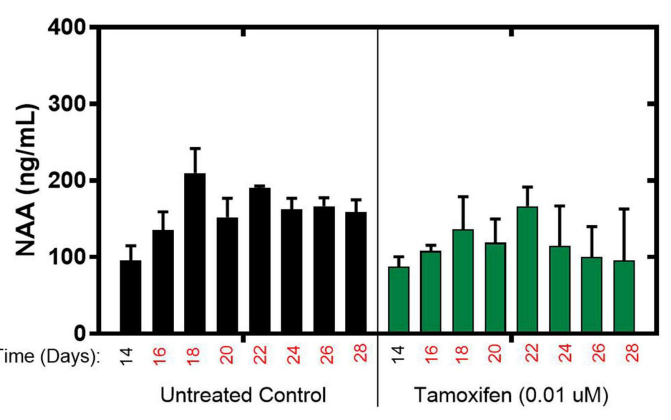

C

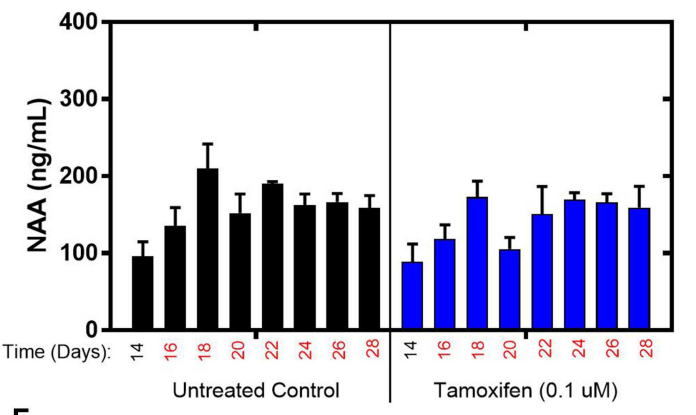

E

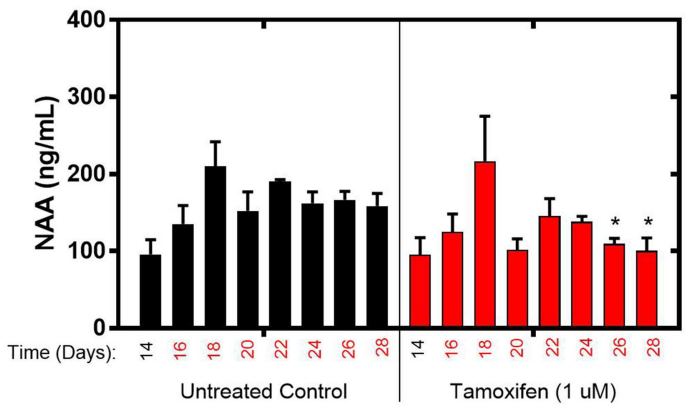

B

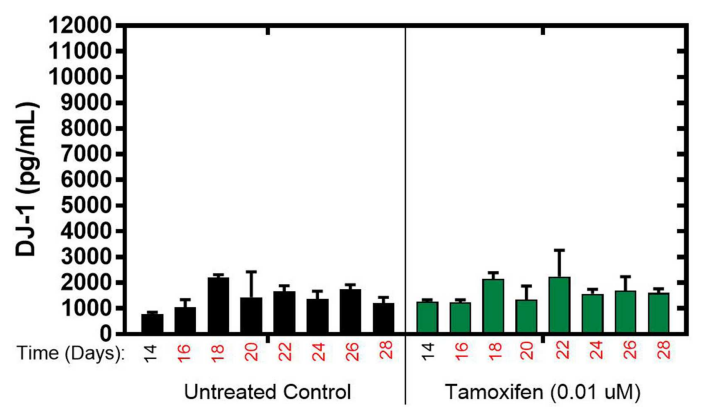

D

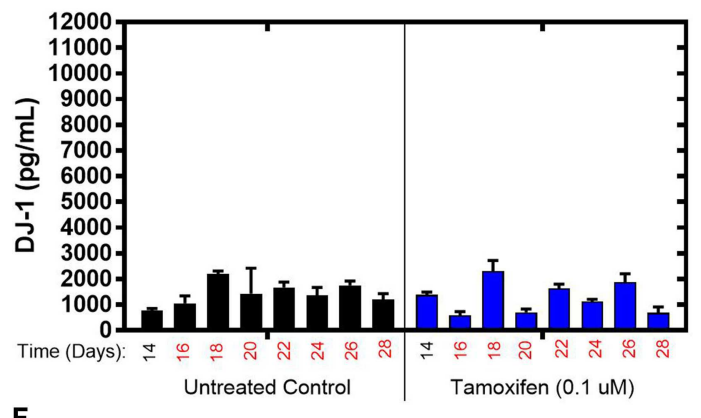

$\mathbf{F}$

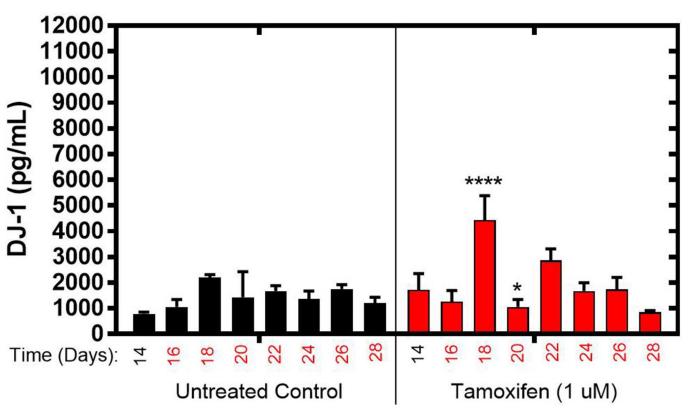

FIGURE 3 | Tamoxifen treatment for 14-days of the brain MPS. (A,C,E) Extracellular N-acetyl-aspartate (NAA) concentration in the media for the noted dose (B,D,F) Extracellular DJ-1/PARK7 concentration in the media for the noted dose. Each bar represents mean \pm SD for triplicate MPS wells $(n=3)$ at each treatment dose and time point. For each tamoxifen dose, significant differences between treated (colored bars) and untreated control (black bars) at the same time point are denoted according to the calculated $p$-values (two-way ANOVA multiple comparisons with a Bonferroni's post-hoc test; ${ }^{*} p<0.05$ and ${ }^{* \star * *} p<0.0001$ ). Day 14 represents the time at which drug dosing was initiated.

decrease upon bortezomib treatment, and remained unaltered in the tamoxifen-treated groups.

\section{Bortezomib-Induced Perturbations in Metabolic Pathways Indicate Oxidative Stress and Disruption of Membrane Integrity}

Pathway analysis using the Ingenuity Pathway Analysis (IPA) software was carried out to examine the importance of altered metabolites in the bortezomib mechanism of action. Only middose and high-dose bortezomib-treated groups were used in comparison to untreated controls, since low-dose bortezomib treatment did not induce significant metabolite alteration.
The pathway analysis revealed various diseases and disorders to be associated with the observed metabolite alterations, with "gastrointestinal disease," and "organismal injury and abnormalities" being common between the mid-dose and highdose treated groups (Figure 6A). "Neurological disease" and "cancer metabolic pathways," the only disease and disorder pathways clearly relevant for the brain MPS, were reported to be associated with the metabolite alterations in the high-dose treatment group.

Bortezomib treatment caused a decrease in secreted levels of SSC and cystine (Figure 5), which are both metabolites in cysteine and methionine metabolism (Figure 6B) that regulates redox homeostasis in brain tissue and neurons (McBean, 2017; Paul et al., 2018). Examination of the pathway diagram indicates 

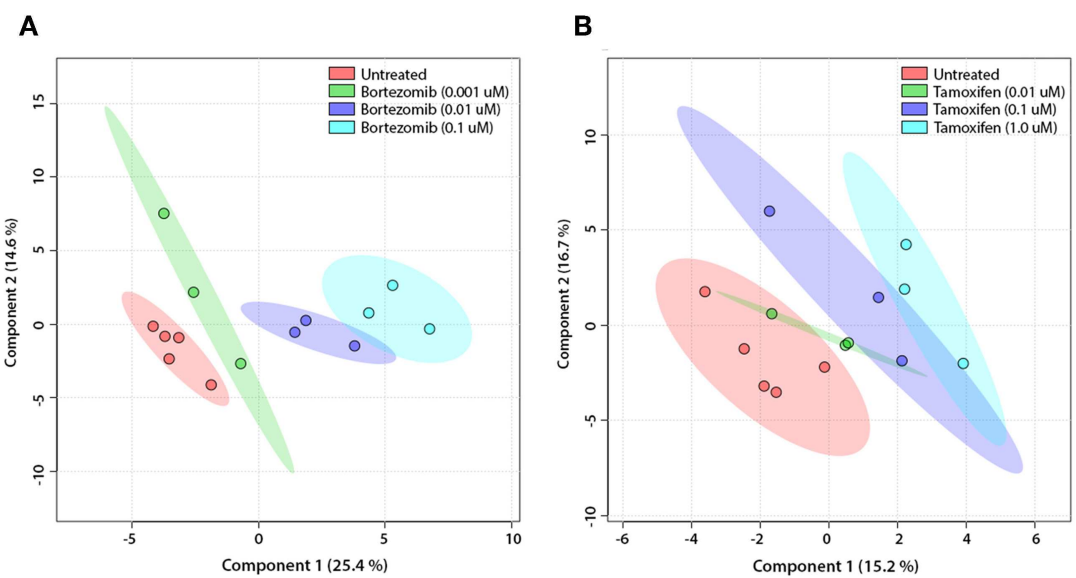

C

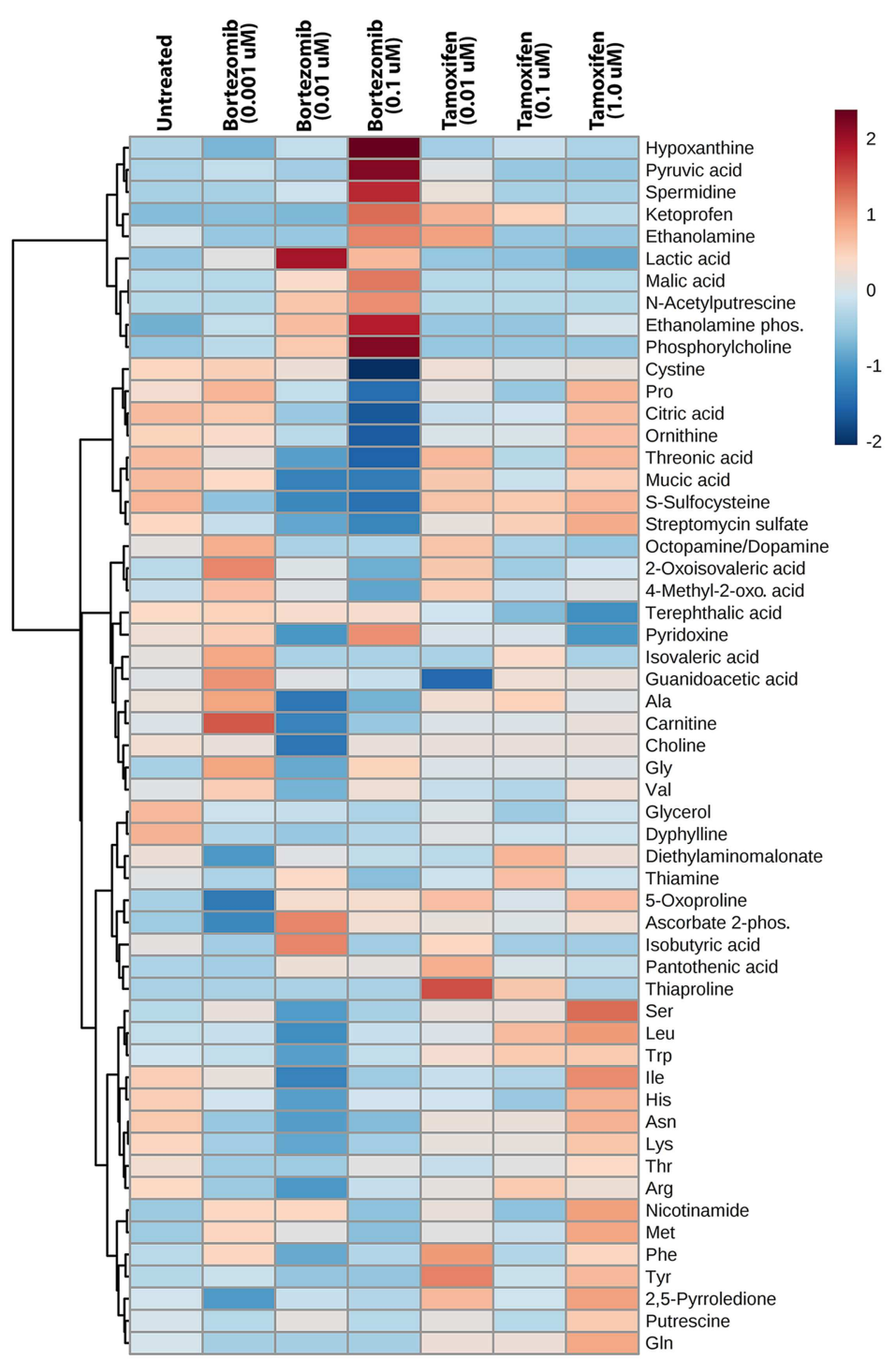

FIGURE 4 | Multivariate and cluster analysis for untargeted metabolomics. PLS-DA scores plots for the (A) bortezomib or (B) tamoxifen treated groups (green, blue, and cyan circles represent low-dose, mid-dose, and high-dose, respectively, with their untreated controls in red circles, all within their $95 \%$ confidence intervals) showing the differences in metabolic fingerprints. (C) Heatmap of relative levels of the detected metabolites showing the untreated group $(n=5)$ compared to bortezomib or tamoxifen treated groups ( $n=3$ for each dose). 
S-Sulfocysteine (SSC)

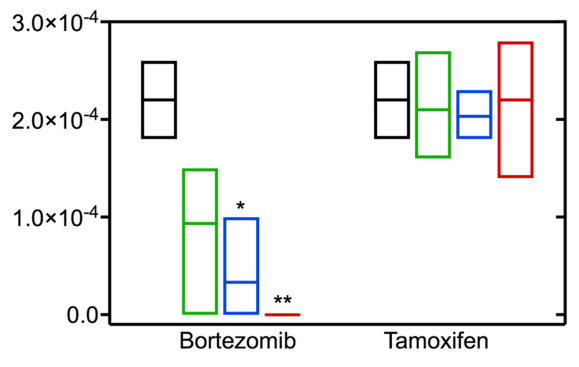

Cystine

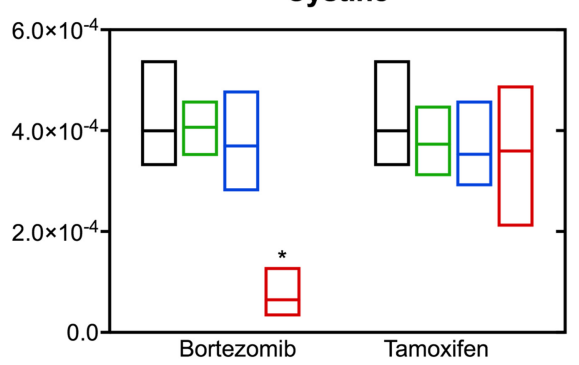

Lactic Acid

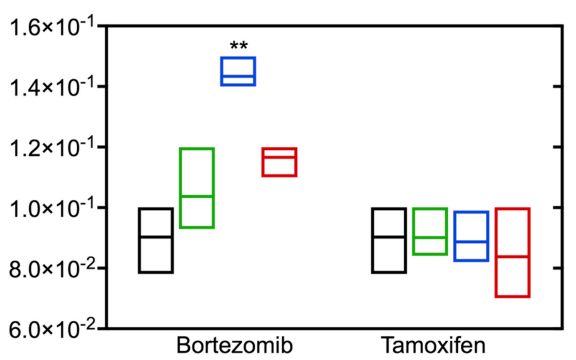

Citric Acid

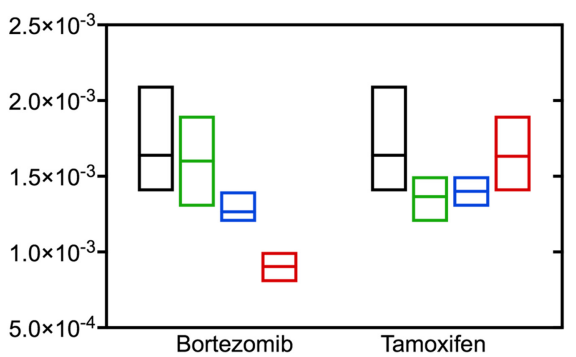

Phosphorylcholine (ChoP)

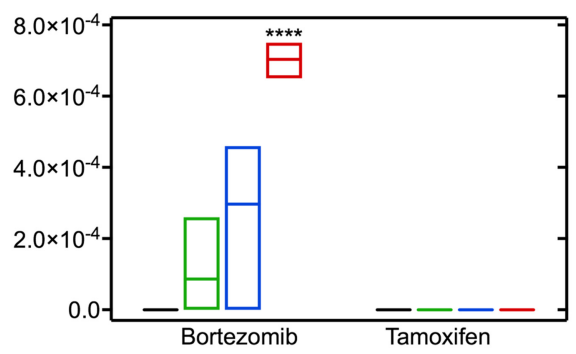

Ethanolamine phosphate (PE)

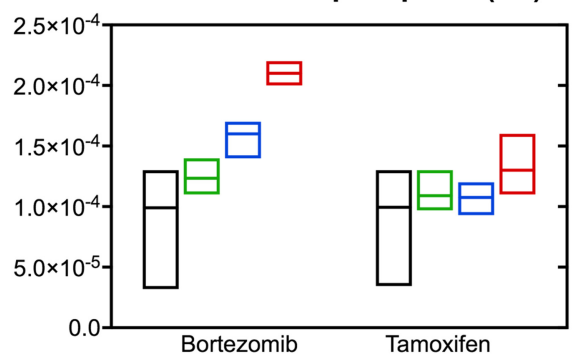

Pyruvate

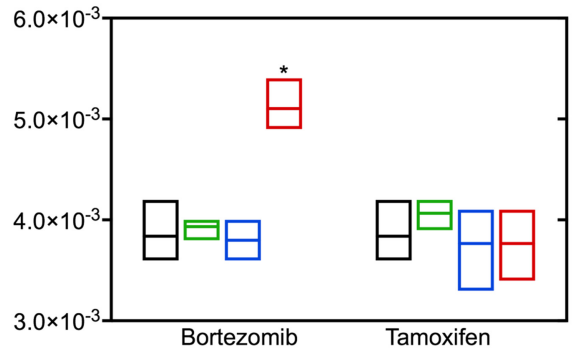

Hypoxanthine

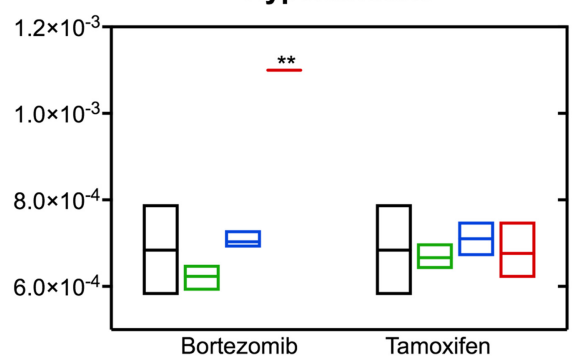

FIGURE 5 | Altered metabolite levels captured 2-days post-treatment with different bortezomib or tamoxifen doses compared to untreated controls. Floating bars (min to max, line at mean) represent relative metabolite abundance for untreated (black, $n=5$ ), low-dose treated (green; $0.001 \mu \mathrm{M}$ bortezomib or $0.01 \mu \mathrm{M}$ tamoxifen, $n=3$ for each drug), mid-dose treated (blue; $0.01 \mu \mathrm{M}$ bortezomib or $0.1 \mu \mathrm{M}$ tamoxifen, $n=3$ for each drug), and high-dose treated (red; $0.1 \mu \mathrm{M}$ bortezomib or $1 \mu \mathrm{M}$ tamoxifen, $n=3$ for each drug) brain MPS. Significant alterations (in drug treated samples, with respect to the untreated controls) are marked according to the adjusted $p$-values (multiple $t$-tests with Holm-Sidak correction; ${ }^{*} p<0.05$, ${ }^{* \star} p<0.01$, and ${ }^{* \star *} p<0.0001$ ). Non-detected metabolite levels are shown at the baseline (as abundance $=0$ ).

relationships among measured metabolites as well as informs the mechanism of action of bortezomib in the brain MPS. These results may suggest a correlation in cystine and SSC levels, and possibly disruption of redox balance in the neural constructs upon bortezomib treatment.
Significant elevations in the levels of extracellular phosphorylcholine (ChoP) and ethanolamine phosphate (PE) in bortezomib-treated groups (Figure 5) were also observed. PE and ChoP are in the glycerophospholipids pathway and both showed similar dose-responses to bortezomib treatment. ChoP is 


\section{A}

\begin{tabular}{|c|c|c|c|}
\hline \multicolumn{4}{|l|}{ Bortezomib - mid-dose } \\
\hline Diseases and Disorders & p-value & \# Molecules & Associated Metabolites \\
\hline Gastrointestinal Disease & 2.89E-02 - 1.14E-05 & 4 & choline, Ile, Leu, carnitine \\
\hline Inflammatory Disease & 4.91E-02 - 1.14E-05 & 4 & choline, Ile, Leu, carnitine \\
\hline Organismal Injury and Abnormalities & 4.91E-02 - 1.14E-05 & 5 & choline, Ile, Leu, carnitine, Asn \\
\hline Cardiovascular Disease & 2.48E-02 - 2.94E-03 & 3 & choline, carnitine, Asn \\
\hline Hematological Disease & 1.66E-02 - 2.94E-03 & 2 & carnitine, Asn \\
\hline \multicolumn{4}{|l|}{ Bortezomib - high-dose } \\
\hline Diseases and Disorders & p-value & \# Molecules & Associated Metabolites \\
\hline Neurological Disease & 4.38E-02 - 5.78E-04 & 5 & citrate, cystine, pyruvate, lactate, $\underline{\mathrm{PE}}$ \\
\hline Cancer & 4.38E-02 - 6.34E-04 & 5 & citrate, Ile, pyruvate, $\mathrm{ChoP}, \underline{\mathrm{PE}}$ \\
\hline Organismal Injury and Abnormalities & 4.38E-02 - 6.34E-04 & 9 & citrate, cystine, Ile, pyruvate, lactate, $\underline{\text { ChoP, }} \underline{\mathrm{PE}}, \underline{\text { hypoxanthine, ketoprofen }}$ \\
\hline Gastrointestinal Disease & 1.66E-02 - 1.09E-03 & 8 & citrate, cystine, Ile, pyruvate, $\underline{\text { ChoP }}, \underline{\mathrm{PE}}$, hypoxanthine, ketoprofen \\
\hline Heredity Disorder & 1.66E-02 - 1.26E-03 & 4 & citrate, cystine, pyruvate, $\mathrm{PE}$ \\
\hline
\end{tabular}

B

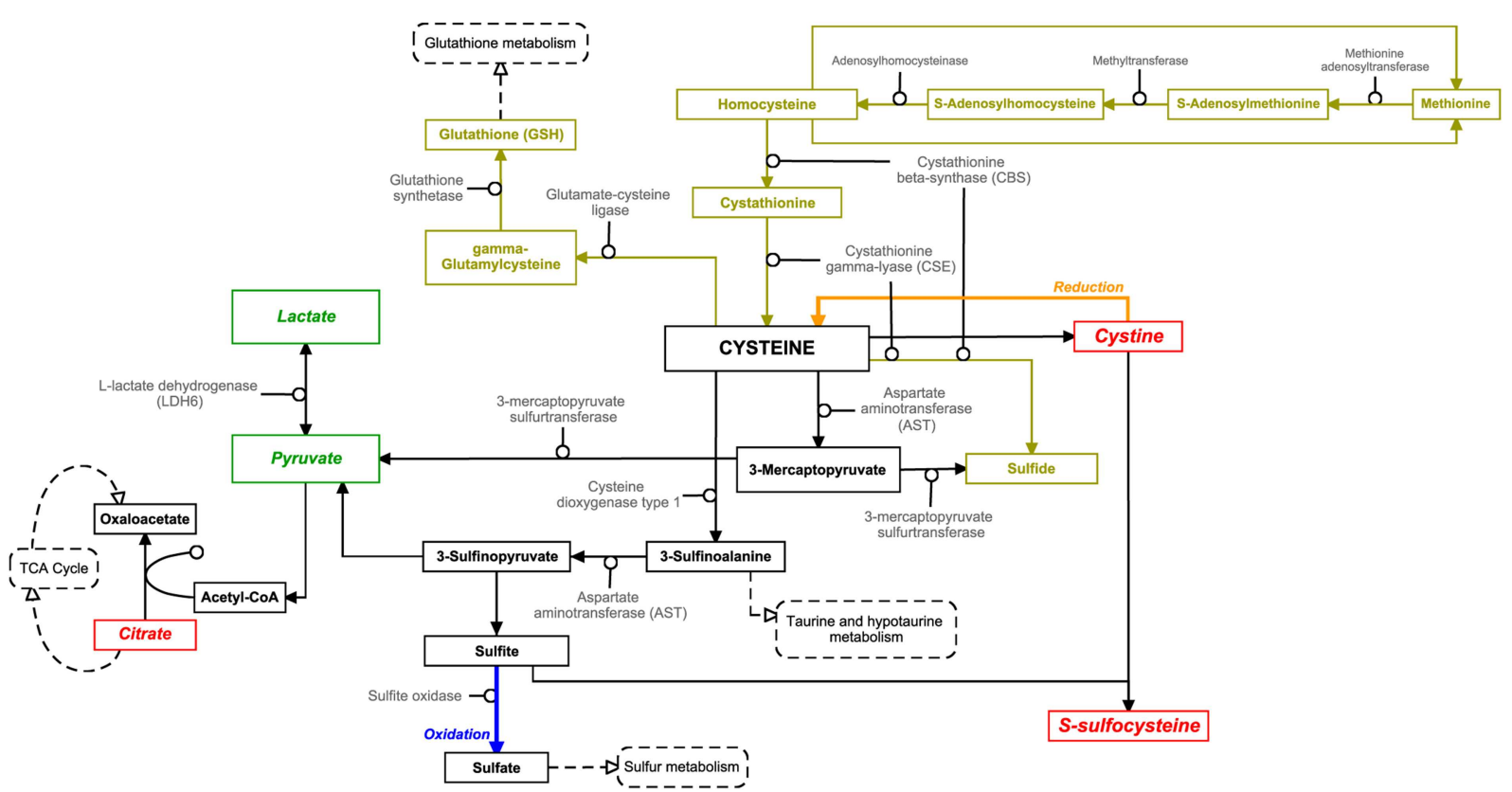

FIGURE 6 | Bortezomib-induced alterations in metabolic pathways. (A) Top diseases and disorders list from Ingenuity Pathway Analysis (IPA) for bortezomib mid-dose, and high-dose treatment. Associated decrease or increase in the metabolites are shown in italic or underlined, respectively. (B) Bortezomib-induced alterations in the cystine and methionine metabolism. Other metabolic pathways connected to cysteine metabolism are shown in dashed rectangles. Solid rectangles represent metabolites in the pathways and the detected metabolites in this study are shown in italic. Transsulfuration pathway (olive color) shows cysteine synthesis from methionine followed by conversion of cysteine to GSH. Bortezomib-induced decrease or increase in extracellular metabolite levels are highlighted in red and green, respectively.

a functional group in the hydrophilic head of the phospholipids forming lipid bilayers, and its release is only possible through a leaky membrane (Walter et al., 2004). Detection of ChoP in the culture media of bortezomib-treated group may suggest that this a consequence of bortezomib-induced disruption of membrane integrity.

\section{Metabolic Signatures Correlate With NAA as Early Indicators of Cellular Dysfunction Followed by DJ-1 Release}

Drug-induced response was observed in metabolic signatures and NAA levels 2-days post treatment. While no alterations in
DJ-1 levels were detected that quickly, after 4-days of bortezomib treatment, DJ-1 levels were in fact upregulated and NAA levels downregulated.

To relate metabolic signatures from untargeted extracellular metabolomics to the clinically-relevant protein biomarkers, correlation analyses were carried out. For all untreated, bortezomib-treated, and tamoxifen-treated groups, NAA, and DJ-1 levels were analyzed with respect to metabolomics measured on day 16 (2-day drug exposure for drug-treated groups). NAA showed both strong positive and negative correlations with many metabolites (Supplementary Table 3 and Supplementary Figure 2), as expected, since NAA, the neuronal health biomarker is a metabolite in alanine, 
aspartate, and glutamate metabolism. To verify the NAAmetabolite correlations, a linear regression model was used for each metabolite with correlation coefficient higher than $0.7(|r|>0.7)$ and a $p$-value lower than 0.05 (Supplementary Table 3, metabolites in bold); the regression line with $95 \%$ confidence interval was mapped over the scatter plot (Supplementary Figure 3).

Similar analyses carried out for DJ-1 (measured on day 16) showed that this oxidative stress biomarker did not have any strong correlations with the metabolic fingerprints on the same time point (Supplementary Table 3 and Supplementary Figure 2). Considering the measured DJ-1 levels on day 18 recapitulated the drug-response more precisely, correlation analyses were also carried out with accumulated DJ-1 levels (total amount measured on days 16 and 18). Interestingly, accumulated DJ-1 was strongly correlated with the metabolites that showed strong correlations with NAA on day 16 (Supplementary Figures 2, 4 and Supplementary Table 3).

These results show that the metabolic signatures and NAA levels correlate well with the measured DJ-1 levels over the longer time period. This finding suggests that a subset of metabolites along with NAA could predict the later DJ-1 levels; the phenotypic outcome indicating cell death induced by cellular dysfunction associated with oxidative stress. To detect this subset, Lasso, a regularized regression method was employed to select the predictors (metabolites) that best define the dependent variable, accumulated DJ-1. The final model constructed at the optimized tuning parameter $(\lambda)$, selected ethanolamine phosphate (PE), hypoxanthine, and phosphorylcholine (ChoP) as the predictors while filtering out the rest of the metabolites that are not of importance for prediction (Supplementary Table 4 and Supplementary Figure 5). Using this model, accumulated DJ-1 was predicted, and the goodness of fit $\left(R^{2}\right)$ of the prediction was calculated as 0.88 with respect to the measured DJ-1 levels (Figure 7). These results indicate that the measured DJ-1 at the later time point could be predicted using the measured relative abundance values of this subset of metabolites on the earlier time point.

\section{DISCUSSION}

Early assessment of adverse effects of therapeutic drugs in humans is crucial to avoid long-lasting harm. The detection of adverse phenotypic responses of tissues and organs demands continuous monitoring of response biomarkers in humans. Metabolomic biomarkers have the potential to provide early insights about organ- and tissue-level dysfunction prior to irreversible toxicity occurring. In this regard, human MPS technologies, which are designed to be more physiologicallyrelevant than existing $2 \mathrm{D}$ cellular in vitro systems, may provide preclinical platforms for drug screening studies. These technologies support long-term tissue culture, enabling the study of kinetics of drug-physiology interactions using various continuous and endpoint metrics, such as clinical biomarkers and -omics. As such, for toxicology, these technologies hold promise not only for identifying toxic effects at the preclinical

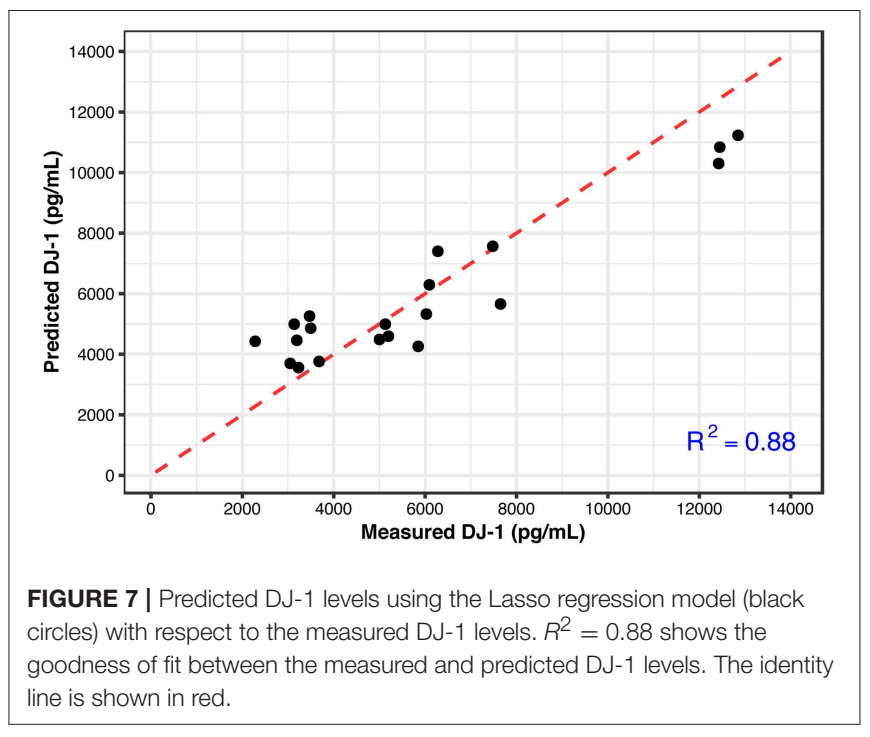

stage but also providing insights about toxicity mechanisms, identification of biomarkers, and informing clinical biomarker strategies. Similar to the existing 2D in vitro models, utilization of the MPS technologies also necessitates assay development such as biomarkers, cell viability/metabolism, and cytotoxicity, in terms of media interference of the assay on detection, assay sensitivity, and molecular stability. Additionally, accurate comparison of in vitro systems requires quantitative characterization of each experimental system, e.g., cell population, cell numbers, effect of different medium composition on in vitro drug bioavailability, and accuracy of the analytical methods.

This study focuses on drug-induced neurotoxicity using a brain microphysiological system (MPS) treated with known neurotoxic and non-neurotoxic drugs, and assessment using both targeted and untargeted molecular profiling. The untreated brain MPS was characterized using immunocytochemistry imaging and measurement of targeted biomarkers (NAA and DJ-1) over the 28-day cell culture. The immunocytochemistry imaging demonstrated similar mixed neuron-glial cell culture extending around the circumference on days 14 and 28 for untreated controls. Additionally, over the 28-day culture period, NAA and DJ-1 levels gradually increased from the start of the cell culture and reached a plateau after day 14 . The drug responses were then evaluated between days 14 and 28 using targeted and untargeted biomarkers.

Enhanced NAA levels were previously found in neuropathological conditions, suggesting that measurement of NAA could be means of monitoring neuronal health (Simone et al., 2011; Tortorella et al., 2011). In this study, 2-days of bortezomib treatment induced high levels of NAA release and altered the cells' metabolic fingerprint, suggesting cellular dysfunction. After 4-days of drug treatment, high levels of DJ-1 were also observed, confirming bortezomib-induced oxidative stress. This sequence suggests that neuronal function is impaired quickly, as indicated by NAA, even while overt oxidative stress damaging the cell membrane as indicated by DJ-1 leakage into the medium is only measurable several days later. The untargeted metabolomics 
results further support these findings, with the elevated levels of PE, a phospholipid breakdown product, and ChoP, a compound released from the cells with leaky membranes. This study suggests that a subset of the detected metabolites in the untargeted metabolomics study following 2-days of treatment could predict the phenotypic outcome (DJ-1) observed at a later time point (after 4-days of treatment). The subset of metabolites that were predictive consisted of ethanolamine phosphate (PE), hypoxanthine, and phosphorylcholine (ChoP). These findings are of particular relevance since NAA and DJ-1, known relevant molecular biomarkers, combined with metabolomics could reveal additional biomarkers to be used in the clinic and could be utilized for assessment of drug-induced neurotoxicity of NMEs.

Bortezomib, used in the treatment of relapsed/refractory multiple myeloma and mantle cell lymphoma, has been reported to induce gastrointestinal toxicity, thrombocytopenia, asthenia, peripheral neuropathy (Menashe, 2007) and have adverse pulmonary effects (Miyakoshi et al., 2006). Exposure to bortezomib causes unfolded proteins to accumulate in the endoplasmic reticulum (ER), causing ER stress and triggering cell stress associated with ROS accumulation (Ri, 2016). Indeed, previous in vitro proteasome inhibition studies showed direct mitochondrial function effect, causing accumulation of ubiquitinated proteins within the mitochondrion and leading to increased reactive oxygen production (Sullivan et al., 2004; Torres and Perez, 2008). In the brain, reactive oxygen and nitrogen species are mostly elevated compared to other organs in the body, and the redox balance would normally be regulated via the antioxidants GSH and cysteine. Disruption of this redox homeostasis has been reported to play an important role in progression of neurodegenerative disorders (Shanker and Aschner, 2001; McBean, 2017; Paul et al., 2018).

In this study, bortezomib had a dose-dependent effect on the levels of the metabolites SSC, and cystine, the oxidized form of cysteine, whereas these were unaltered by tamoxifen treatment. SSC, a product of a not well-established cystinesulfite reaction, was shown to exhibit depolarization (Meweitt et al., 1983), and elevated levels of SSC contributed to neurotoxicity by decreasing intracellular levels of free radicals (Moore et al., 1987). Both SSC and cystine are found in the cysteine metabolism pathway that regulates redox homeostasis in brain tissue and neurons (McBean, 2017; Paul et al., 2018). For the regulation of cysteine metabolism, cells use multiple mechanisms to maintain a constant cysteine supply, either by synthesizing it from methionine via the reverse transsulfuration pathway (Figure 6B), or by the uptake of extracellular cystine via the transporter system $\mathrm{x}_{\mathrm{c}}^{-}$. The observed decrease in the extracellular cystine levels in the highdose bortezomib-treated group may indicate increased cystine uptake from the media (Figure 5 and Supplementary Figure 1). The $\mathrm{x}_{\mathrm{c}}^{-}$system transports extracellular cystine in exchange with glutamate. In this study, extracellular glutamate levels were detected only for the high-dose bortezomib treated group (Supplementary Figure 1), in accordance with the lower extracellular cystine levels. Similar findings were reported suggesting that depletion of extracellular cyst(e)ine triggers oxidative stress via depletion of intracellular GSH, followed by cell cycle arrest and cell death (Cramer et al., 2017).

Oxidative stress or disruption of redox homeostasis in general has been associated with neurodegenerative diseases, due to change in the glutamate levels in the brain, causing calcium influx into neurons, eventually leading to breakdown of membrane structural elements (Klein, 2000). In this context, phospholipids, the essential structural elements of the cell membrane, were reported to be elevated in the cerebrospinal fluid of Alzheimer patients (Walter et al., 2004). Our results in the brain MPS were similar, where bortezomib treatment induced significant elevations in both extracellular ChoP and PE. PE, a precursor of phospholipid synthesis, is a phospholipid breakdown product, and contributes to the sphingolipid metabolism. ChoP is a functional group in the hydrophilic head of the phospholipids forming the lipid bilayers and release of this compound from the cell is possible through a leaky membrane (Walter et al., 2004). These observations may suggest release of ChoP to the culture media is a consequence of bortezomib-induced disruption of membrane integrity. For untreated and tamoxifen treated groups, ChoP was undetectable in the MPS medium. ChoP normally can be hydrolyzed to free choline in the cell and be released through certain transporters (Klein, 2000). However, changes in the extracellular choline levels are not specific to disruption of membrane integrity since choline could be hydrolyzed from (or condensed to) acetylcholine, or oxidized to betaine which provides methyl groups to form methionine in the transsulfuration pathway of the cysteine and methionine metabolism. In this study, a decrease in the extracellular choline levels for the low-dose and mid-dose bortezomib treated groups was observed, but the levels were comparable for the high-dose bortezomib, tamoxifen treated, and untreated groups (Supplementary Figure 1). Hence, an increased ChoP release could be an indicator of membrane breakdown, but not the only driving force of the change in the extracellular choline levels.

Significant changes in the levels of lactic acid, pyruvate, citric acid, and hypoxanthine were detected upon bortezomib treatment but not with tamoxifen. The functions of several of these may give clues to the mechanisms by which bortezomib is adversely affecting the tissue. Hypoxanthine is a purine derivative commonly utilized as a hypoxia biomarker (Saugstad, 1988), while citric acid is a key constituent in the TCA cycle. While the changes observed in the citrate levels may be correlated with pyruvate (and lactate) through the TCA cycle, previous studies reported that citrate is synthesized in and released from astrocytes in large amounts, which contributes to its regulatory function in the CNS as an extracellular chelating agent (Westergaard et al., 2017). Moreover, bortezomib-induced perturbations in these metabolites may also suggest mitochondrial dysfunction of the neural constructs.

For the statistical analysis of the metabolomics data, multiple $t$-tests were carried out and Holm-Sidak correction was applied to account for multiple comparisons problem. The Sidak method allows strong control on the familywise error rate (FWER), and it is believed to be too conservative in some cases. False discovery rate (FDR) measure may be more reasonable since 
it allows a proportion of Type I errors to occur. When the statistical analyses were repeated with FDR measure (with $\mathrm{Q}=5 \%$ ), our results were the same for tamoxifen treatments, and low- and mid-dose bortezomib treatments, while the number of significantly altered metabolites upon high-dose bortezomib treatment were higher (data not shown). Metabolites such as citric acid, and PE, which were not significant in the Holm-Sidak method were significant in the FDR method. Considering that Sidak correction might be too conservative, the metabolites with lower adjusted- $p$-values (0.05-0.1) were already included in our biological interpretation of the metabolomics data.

In summary, clinically relevant biomarkers for neural health and neurotoxic response were quantified in the brain MPS. The changes in biomarker profiles upon neurotoxic drug treatment using both targeted and untargeted molecular biomarkers indicated oxidative stress and membrane breakdown in the brain MPS. Furthermore, a subset of early response biomarkers identified with untargeted metabolomics after a first dose of neurotoxic drug administration was correlated to DJ-1 protein levels, which was observed after repeated drug dosing. These results indicated that early response biomarkers could be used to predict adverse drug effects in repeated drug dosing regimens before overt cell death occurs. These clinically relevant biomarkers could also be used to assess early response in humans to avoid irreversible drug-induced toxicity. While our study does not provide a deep biological characterization of the brain MPS and its relevance to the human brain in terms of tissue/cell content and organ function, we believe this study to be a proof of concept for the assessment of preclinical response biomarkers and utilization of a $3 \mathrm{D}$ in vitro model for possible drug screening studies. Under the conditions used in the study, we found that NAA, DJ-1, and extracellular untargeted metabolomics were able to capture a differential response for a neurotoxic, and a non-neurotoxic drug. It should be noted that response biomarkers are highly dependent on the drug mechanism of action (MoA), and should be chosen with care for large scale screening of drugs that belong to different classes of MoA. We acknowledge the limitations of

\section{REFERENCES}

Argyriou, A. A., Cavaletti, G., Bruna, J., Kyritsis, A. P., and Kalofonos, H. P. (2014). Bortezomib-induced peripheral neurotoxicity: an update. Arch. Toxicol. 88, 1669-1679. doi: 10.1007/s00204-014-1316-5

Badros, A., Goloubeva, O., Dalal, J. S., Can, I., Thompson, J., Rapoport, A. P., et al. (2007). Neurotoxicity of bortezomib therapy in multiple myeloma: a single-center experience and review of the literature. Cancer 110, 1042-1049. doi: $10.1002 / \mathrm{cncr} .22921$

Bittner, D. M., Heinze, H.-J., and Kaufmann, J. (2013). Association of 1HMR spectroscopy and cerebrospinal fluid biomarkers in Alzheimer's disease: diverging behavior at three different brain regions. J. Alzheimers Dis. 36, 155-163. doi: 10.3233/JAD-120778

Canta, A., Pozzi, E., and Carozzi, V. (2015). Mitochondrial dysfunction in Chemotherapy-Induced Peripheral Neuropathy (CIPN). Toxics 3, 198-223. doi: 10.3390/toxics3020198

Chen, W. L. K., Edington, C., Suter, E., Yu, J., Velazquez, J. J., Velazquez, J. G., et al. (2017). Integrated gut/liver microphysiological systems elucidates the brain MPS, sensitivity of the NAA, DJ-1, and untargeted metabolomics measurements, and the drugs used in this study as they provide an individual case study rather than demonstrating a high throughput drug screening or prediction of neurotoxicity. However, we suggest that the approach demonstrated here can be extended for preclinical toxicity screening and biomarker discovery studies.

\section{AUTHOR CONTRIBUTIONS}

WM and JT developed the brain microphysiological system and provided tissue culture protocols. SM and MC planned the experiments. SM performed the experiments, analyzed the data, and wrote the main manuscript text. BA performed the metabolomics analysis and wrote the main manuscript text. CS and MC directed the work and edited the manuscript. All authors reviewed the manuscript.

\section{ACKNOWLEDGMENTS}

Research reported in this publication was supported by the National Center for Advancing Translational Sciences of the National Institutes of Health under Award Number NIHU24TR001951 and NIH-UH3TR000506-03. The content is solely the responsibility of the authors and does not necessarily represent the official views of the National Institutes of Health. Collin Edington and Iris Lee are gratefully acknowledged for the training using the standard operating procedure (SOPs) for cell culture, and Christian Alexander Maass for helpful statistical discussion. We are also very grateful to Alexander Buko and Takushi Oga (Human Metabolome Technologies) for their valuable input and discussions.

\section{SUPPLEMENTARY MATERIAL}

The Supplementary Material for this article can be found online at: https://www.frontiersin.org/articles/10.3389/fdata. 2019.00023/full\#supplementary-material inflammatory inter-tissue crosstalk. Biotechnol. Bioeng. 114, 2648-2659. doi: $10.1002 /$ bit. 26370

Choi, J., Sullards, M. C., Olzmann, J. A., Rees, H. D., Weintraub, S. T., Bostwick, D. E., et al. (2006). Oxidative damage of DJ-1 is linked to sporadic Parkinson and Alzheimer diseases. J. Biol. Chem. 281, 10816-10824. doi: 10.1074/jbc.M509079200

Chong, J., Soufan, O., Li, C., Caraus, I., Li, S., Bourque, G., et al. (2018). MetaboAnalyst 4.0: towards more transparent and integrative metabolomics analysis. Nucleic Acids Res. 46, W486-W494. doi: 10.1093/nar/gky310

Cook, D., Brown, D., Alexander, R., March, R., Morgan, P., Satterthwaite, G., et al. (2014). Lessons learned from the fate of AstraZeneca's drug pipeline: a five-dimensional framework. Nat. Rev. Drug Discov. 13, 419-431. doi: $10.1038 / \mathrm{nrd} 4309$

Cramer, S. L., Saha, A., Liu, J., Tadi, S., Tiziani, S., Yan, W., et al. (2017). Systemic depletion of L-cyst(e)ine with cyst(e)inase increases reactive oxygen species and suppresses tumor growth. Nat. Med. 23, 120-127. doi: 10.1038/nm.4232

Edington, C. D., Chen, W. L. K., Geishecker, E., Kassis, T., Soenksen, L. R., Bhushan, B. M., et al. (2018). Interconnected microphysiological 
systems for quantitative biology and pharmacology studies. Sci. Rep. 8:4530. doi: 10.1038/s41598-018-22749-0

Ernst, T., Chang, L., Cooray, D., Salvador, C., Jovicich, J., Walot, I., et al. (2002). The effects of tamoxifen and estrogen on brain metabolism in elderly women. CancerSpectrum Knowl. Environ. 94, 592-597. doi: 10.1093/jnci/94. 8.592

Fairbanks, B. D., Schwartz, M. P., and Halevi, A. E. (2014). A versatile synthetic extracellular matrix mimic via thiol- norbornene photopolymerization. Sci. Adv. Mater. 21, 5005-5010. doi: 10.1002/adma.200901808

Friedman, J., Hastie, T., and Tibshirani, R. (2010). Regularization paths for generalized linear models via coordinate descent. J. Stat. Softw. 33, 1-22. doi: 10.18637/jss.v033.i01

Hansen, T. D., Koepsel, J. T., Le, N. N., Nguyen, E. H., Zorn, S., Parlato, M., et al. (2014). Biomaterial arrays with defined adhesion ligand densities and matrix stiffness identify distinct phenotypes for tumorigenic and nontumorigenic human mesenchymal cell types. Biomater. Sci. 2, 745-756. doi: 10.1039/C3BM60278H

Harris, K., Lin, A., Bhattacharya, P., Tran, T., Wong, W., and Ross, B. (2006). "Regulation of NAA-synthesis in the human brain in vivo : Canavan's Disease, Alzheimer's Disease and Schizophrenia," in N-Acetylaspartate A Unique Neuronal Molecule in the Central Nervous System, eds J. R. Moffett, S. B. Tieman, D. R. Weinberger, J. T. Coyle, and A. M. A. Namboodiri (Boston, MA: Springer), 263-273.

Hong, C.-T., Chan, L., Hu, C.-J., Lin, C.-M., Hsu, C.-Y., and Lin, M.-C. (2017). Tamoxifen and the risk of Parkinson's disease in female patients with breast cancer in asian people: a nationwide population-based study. J. Breast Cancer 20, 356-360. doi: 10.4048/jbc.2017.20.4.356

Kahle, P., Waak, J., and Gasser, T. (2009). DJ-1 and prevention of oxidative stress in Parkinson's disease and other age-related disorders. Free Radic. Biol. Med. 47, 1354-1361. doi: 10.1016/j.freeradbiomed.2009.08.003

Kim, S. J., Park, Y. J., Hwang, I. Y., Youdim, M. B. H., Park, K. S., and Oh, Y. J. (2012). Nuclear translocation of DJ-1 during oxidative stress-induced neuronal cell death. Free Radic. Biol. Med. 53, 936-950. doi: 10.1016/j.freeradbiomed.2012.05.035

Kisanga, E. R., Gjerde, J., Guerrieri-Gonzaga, A., Pigatto, F., Pesci-Feltri, A., Robertson, C., et al. (2004). Tamoxifen and metabolite concentrations in serum and breast cancer tissue during three dose regimens in a randomized preoperative trial. Clin. Cancer Res. 10, 2336-2343. doi: 10.1158/1078-0432.CCR-03-0538

Klein, J. (2000). Membrane breakdown in acute and chronic neurodegeneration: focus on choline-containing phospholipids. J. Neural Transm. 107, 1027-1063. doi: 10.1007/s007020070051

Krämer, A., Green, J., Pollard, J., and Tugendreich, S. (2014). Causal analysis approaches in ingenuity pathway analysis. Bioinformatics 30, 523-530. doi: 10.1093/bioinformatics/btt703

Kuhn, M. (2008). Building predictive models in R using the caret package. J. Stat. Softw. 28, 1-26. doi: 10.18637/jss.v028.i05

Kutmon, M., van Iersel, M. P., Bohler, A., Kelder, T., Nunes, N., Pico, A. R., et al. (2015). PathVisio 3: an extendable pathway analysis toolbox. PLoS Comput. Biol. 11, 1-13. doi: 10.1371/journal.pcbi. 1004085

Langhans, S. A. (2018). Three-dimensional in vitro cell culture models in drug discovery and drug repositioning. Front. Pharmacol. 9:6. doi: 10.3389/fphar.2018.00006

Low, L. A., and Tagle, D. A. (2017). Microphysiological systems ("Organs-onChips”) for drug efficacy and toxicity testing. Clin. Transl. Sci. 10, 237-239. doi: $10.1111 /$ cts. 12444

Marx, U., Andersson, T. B., Bahinski, A., Beilmann, M., Beken, S., Cassee, F. R., et al. (2016). Biology-inspired microphysiological system approaches to solve the prediction dilemma of substance testing. ALTEX Transatl. Think Tank Toxicol. 33, 272-321. doi: 10.14573/altex.1603161

Maschmeyer, I., Lorenz, A. K., Schimek, K., Hasenberg, T., Ramme, A. P., Hübner, J., et al. (2015). A four-organ-chip for interconnected long-term co-culture of human intestine, liver, skin and kidney equivalents. Lab Chip 15, 2688-2699. doi: 10.1039/C5LC00392J

McBean, G. (2017). Cysteine, glutathione, and thiol redox balance in astrocytes. Antioxidants 6:62. doi: 10.3390/antiox6030062

Menashe, J. (2007). Managing and avoiding bortezomib toxicity. Commun. Oncol. 4, 480-484. doi: 10.1016/S1548-5315(11)70481-8
Meweitt, K., Oakes, D., Olverman, H., Smith, D., and Watkins, J. (1983). Pharmacology of the excitatory actions of suphonic and sulphinic amino acids. Adv. Biochem. Psychopharmacol. 37, 163-174.

Miyakoshi, S., Kami, M., Yuji, K., Matsumura, T., Takatoku, M., Sasaki, M., et al. (2006). Brief report Severe pulmonary complications in Japanese patients after bortezomib treatment for refractory multiple myeloma. Blood 107, 3492-3494. doi: 10.1182/blood-2005-11-4541

Moffett, J. R., Tieman, S. B., Weinberger, D. R., Coyle, J. T., and Naamboodiri, A. M. A. (2012). N-Acetylaspartate: A Unique Neuronal Molecule in the Central Nervous System. New York, NY: Springer.

Moore, W., Wiener, H. L., and Meister, A. (1987). Inactivation of yglutamylcysteine synthetase, but not of glutamine synthetase, by s-sulfocysteine and s-sulfohomocysteine. J. Biol. Chem. 262, 16771-16777.

Moreau, P., Karamanesht, I. I., Domnikova, N., Kyselyova, M. Y., Vilchevska, K. V., Doronin, V. A., et al. (2012). Pharmacokinetic, pharmacodynamic and covariate analysis of subcutaneous versus intravenous administration of bortezomib in patients with relapsed multiple myeloma. Clin. Pharmacokinet. 51, 823-829. doi: 10.1007/s40262-012-0010-0

Murray, M. E., Przybelski, S. A., Lesnick, T. G., Liesinger, A. M., Spychalla, A., Zhang, B., et al. (2014). Early Alzheimer's disease neuropathology detected by proton MR spectroscopy. J. Neurosci. 34, 16247-16255. doi: 10.1523/JNEUROSCI.2027-14.2014

Oleaga, C., Bernabini, C., Smith, A. S. T., Srinivasan, B., Jackson, M., McLamb, W., et al. (2016). Multi-organ toxicity demonstration in a functional human in vitro system composed of four organs. Sci. Rep. 6:20030. doi: 10.1038/srep 20030

Paul, B. D., Sbodio, J. I., and Snyder, S. H. (2018). Cysteine metabolism in neuronal redox homeostasis. Trends Pharmacol. Sci. 39, 513-524. doi: 10.1016/j.tips.2018.02.007

Poste, G. (2011). Bring on the biomarkers. Nature 469, 156-157. doi: $10.1038 / 469156 a$

Ri, M. (2016). Mechanism of action of bortezomib in multiple myeloma therapy. Int. J. Myeloma 6, 1-6. Available online at: http://www.jsm.gr.jp/files/ journalpdf/2016_6_1_ri-final.pdf

Saugstad, O. D. (1988). Hypoxanthine as an indicator of hypoxia: its role in health and disease through free radical production. Pediatr. Res. 23, 143-150. doi: 10.1203/00006450-198802000-00001

Schultz, L., Zurich, M.-G., Culot, M., da Costa, A., Landry, C., Bellwon, P., et al. (2015). Evaluation of drug-induced neurotoxicity based on metabolomics, proteomics and electrical activity measurements in complementary CNS in vitro models. Toxicol. Vitro 30, 138-165. doi: 10.1016/j.tiv.2015. 05.016

Schwartz, M. P., Hou, Z., Propson, N. E., Zhang, J., Engstrom, C. J., Costa, V. S., et al. (2015). Human pluripotent stem cell-derived neural constructs for predicting neural toxicity. Proc. Natl. Acad. Sci. U S A. 112, 12516-12521. doi: 10.1073 /pnas. 1516645112

Shanker, G., and Aschner, M. (2001). Identification and characterization of uptake systems for cystine and cysteine in cultured astrocytes and neurons: evidence for methylmercury-targeted disruption of astrocyte transport. J. Neurosci. Res. 66, 998-1002. doi: 10.1002/jnr.10066

Simone, I. L., Ruggieri, M., Tortelli, R., Ceci, E., D’Errico, E., Leo, A., et al. (2011). Serum N-acetylaspartate level in amyotrophic lateral sclerosis. Arch. Neurol. 68, 1308-1312. doi: 10.1001/archneurol.2011.217

Stouten-Kemperman, M. M., De Ruiter, M. B., Koppelmans, V., Boogerd, W., Reneman, L., and Schagen, S. B. (2015). Neurotoxicity in breast cancer survivors $\geq 10$ years post-treatment is dependent on treatment type. Brain Imaging Behav. 9, 275-284. doi: 10.1007/s11682-014-9305-0

Sullivan, P. G., Dragicevic, N. B., Deng, J. H., Bai, Y., Dimayuga, E., Ding, Q., et al. (2004). Proteasome inhibition alters neural mitochondrial homeostasis and mitochondria turnover. J. Biol. Chem. 279, 20699-20707. doi: 10.1074/jbc.M313579200

Tibshirani, R. (1996). Regression shrinkage and selection via the lasso. J. R. Stat. Soc. Ser. B 58, 267-288. doi: 10.1111/j.2517-6161.1996.tb02080.x

Torres, C. A., and Perez, V. I. (2008). Proteasome modulates mitochondrial function during cellular senescence. Free Radic. Biol. Med. 44, 403-414. doi: 10.1016/j.freeradbiomed.2007.10.002

Tortorella, C., Ruggieri, M., Di Monte, E., Ceci, E., Iaffaldano, P., Direnzo, V., et al. (2011). Serum and CSF N-acetyl aspartate levels differ in multiple sclerosis 
and neuromyelitis optica. J. Neurol. Neurosurg. Psychiatry 82, 1355-1359. doi: 10.1136/jnnp.2011.241836

Tsamandouras, N., Chen, W. L. K., Edington, C. D., Stokes, C. L., Griffith, L. G., and Cirit, M. (2017). Integrated gut and liver microphysiological systems for quantitative in vitro pharmacokinetic studies. AAPS J. 19, 1499-1512. doi: 10.1208/s12248-017-0122-4

Tsamandouras, N., Kostrzewski, T., Stokes, C. L., Griffith, L. G., Hughes, D. J., and Cirit, M. (2016). Quantitative assessment of population variability in hepatic drug metabolism using a perfused three-dimensional human liver microphysiological system. J. Pharmacol. Exp. Ther. 360, 95-105. doi: $10.1124 /$ jpet.116.237495

Walker, A. L., Imam, S. Z., and Roberts, R. A. (2018). Drug discovery and development: biomarkers of neurotoxicity and neurodegeneration. Exp. Biol. Med. 243, 1037-1045. doi: 10.1177/1535370218 801309

Walter, A., Korth, U., Hilgert, M., Hartmann, J., Weichel, O., Hilgert, M., et al. (2004). Glycerophosphocholine is elevated in cerebrospinal fluid of Alzheimer patients. Neurobiol. Aging 25, 1299-1303. doi: 10.1016/j.neurobiolaging.2004.02.016

Westergaard, N., Waagepetersen, H. S., Belhage, B., and Schousboe, A. (2017). Citrate, a ubiquitous key metabolite with regulatory function in the CNS. Neurochem. Res. 42, 1583-1588. doi: 10.1007/s11064-0162159-7
Wittsack, H. J., Kugel, H., Roth, B., and Heindel, W. (1996). Quantitative measurements with localized $1 \mathrm{H}$ MR spectroscopy in children with Canavan's disease. J. Magn. Reson. Imaging 6, 889-893. doi: 10.1002/jmri.1880060609

Zhang, Y. S., Aleman, J., Shin, S. R., Kilic, T., Kim, D., Mousavi Shaegh, S. A., et al. (2017). Multisensor-integrated organs-on-chips platform for automated and continual in situ monitoring of organoid behaviors. Proc. Natl. Acad. Sci. U S A. 114, E2293-E2302. doi: 10.1073/pnas.1612906114

Conflict of Interest Statement: WM is a co-founder and stockholder in Stem Pharm, Inc. MC is a co-founder and stockholder in Javelin Biotech, Inc. CS is employed by the company Stokes Consulting.

The remaining authors declare that the research was conducted in the absence of any commercial or financial relationships that could be construed as a potential conflict of interest.

Copyright (๑ 2019 Mina, Alaybeyoglu, Murphy, Thomson, Stokes and Cirit. This is an open-access article distributed under the terms of the Creative Commons Attribution License (CC BY). The use, distribution or reproduction in other forums is permitted, provided the original author $(s)$ and the copyright owner(s) are credited and that the original publication in this journal is cited, in accordance with accepted academic practice. No use, distribution or reproduction is permitted which does not comply with these terms. 